\title{
SNR Study of Outdoor Electroluminescence Images under High Sun Irradiation
}

Mantel, Claire; Benatto, Gisele Alves dos Reis; Riedel, Nicholas; Thorsteinsson, Sune; Poulsen, Peter Behrensdorff; Parikh, Harsh; Spataru, Sergiu; Sera, Dezso; Forchhammer, Søren

\section{Published in:}

Proceedings of 7th World Conference on Photovoltaic Energy Conversion

Link to article, DOI:

10.1109/PVSC.2018.8548264

Publication date:

2018

Document Version

Peer reviewed version

Link back to DTU Orbit

Citation (APA):

Mantel, C., Benatto, G. A. D. R., Riedel, N., Thorsteinsson, S., Poulsen, P. B., Parikh, H., Spataru, S., Sera, D., \& Forchhammer, S. (2018). SNR Study of Outdoor Electroluminescence Images under High Sun Irradiation. In Proceedings of 7th World Conference on Photovoltaic Energy Conversion (pp. 3285-3289). IEEE.

https://doi.org/10.1109/PVSC.2018.8548264

\section{General rights}

Copyright and moral rights for the publications made accessible in the public portal are retained by the authors and/or other copyright owners and it is a condition of accessing publications that users recognise and abide by the legal requirements associated with these rights.

- Users may download and print one copy of any publication from the public portal for the purpose of private study or research.

- You may not further distribute the material or use it for any profit-making activity or commercial gain

- You may freely distribute the URL identifying the publication in the public portal 


\title{
SNR Study of Outdoor Electroluminescence Images under High Sun Irradiation
}

\author{
Claire Mantel ${ }^{1}$, Gisele A. dos Reis Benatto ${ }^{1}$, Nicholas Riedel ${ }^{1}$, Sune Thorsteinsson ${ }^{1}$, Peter B. Poulsen ${ }^{1}$, \\ Harsh Parikh², Sergiu Spataru ${ }^{2}$, Dezso Sera ${ }^{2}$ and Søren Forchhammer ${ }^{1}$ \\ ${ }^{1}$ Department of Photonics Engineering, Technical University of Denmark, Frederiksborgvej 399, 4000, \\ Roskilde, Denmark \\ ${ }^{2}$ Aalborg University, Pontoppidanstraede 101, 9220, Aalborg, Denmark
}

\begin{abstract}
Electroluminescence (EL) imaging inspections of $P V$ power plants can bring a huge improvement in accuracy. The use of InGaAs camera will also make such inspections fast, but the restriction to acquire the images during dusk or evening is a limitation. Performing lock-in EL is a way to go for daylight EL. This paper proposes an extension of the $\mathrm{SNR}_{50}$ quality measure to estimate the quality of a stack of $N$ images and evaluates the impact of some factors over the measured and visual quality of images acquired with InGaAs sensors. The factors analyzed are the characteristics of the noise in the acquired images, the influence of the sun variations and the averaging over multiple acquired images.
\end{abstract}

\section{INTRODUCTION}

Electroluminescence imaging can be used to rapid and accurately detect a large range of major and minor faults in PV modules, unlike the most commonly used techniques for large scale PV inspection. The faults entitle to be detected with EL include Potential Induced Degradation (PID), cell cracks, broken busbars and interconnections, among others [1], [2]. Due to price, accessibility and high resolution, the most commonly used camera for EL have silicon-based detectors (such as CCD), which present a weak spectral sensitivity in the EL emission range of crystalline silicon solar cells. Their long integration times (in the order of seconds) directly limit inspection speed and their application in unmanned aerial vehicle (UAV) face crucial technical challenges. Cameras with InGaAs-based detectors have a good spectral response to the silicon luminescence emission and therefore short integration times, which can be even shorter than $1 \mathrm{~ms}$ [3].

A signal-to-noise ratio (SNR) quality criteria is established by the EL standard [4] as $\mathrm{SNR}_{50}$ : it is performed on three images (two with bias current applied to the panel and one of unpowered module). It is computed as:

$$
S N R_{50}=\frac{\sum_{k}\left(0.5 \cdot\left(E L_{1}(k)+E L_{2}(k)\right)-B G(k)\right)}{\sum_{k}\left(\left|E L_{1}(k)-E L_{2}(k)\right| \cdot \sqrt{0.5} \cdot\left(\frac{2}{\pi}\right)^{-0.5}\right)}
$$

where $k$ is the pixel index, $\mathrm{EL}_{1}$ and $\mathrm{EL}_{2}$ two biased images and BG one image acquired with unpowered module.
For daylight outdoor imaging, a set of two or three images is too noisy. However, outdoor EL under sunlight can be performed with the application of synchronized electrical signal and camera triggering and acquisition of more than 3 images alternating between each mode [5]-[8]. A "de-noised" image $E L_{A V G}$ is then obtained by filtering all the biased images, for example by averaging them. The same procedure is applied to create a denoised unbiased image $B G_{A V G}$. Finally, the difference image is computed Diff $A V G=E L_{A V G}-B G_{A V G}$ and used to evaluate the defects on the considered panel. Therefore, for daylight outdoor imaging, there is a need for evaluating the quality of the final Diff ${ }_{A V G}$ obtained from a stack of images, which is not covered by the $\mathrm{SNR}_{50}$.

In this paper, we propose an extension of the $\mathrm{SNR}_{50}$ to the case of multiple images acquired as a sequence. The paper is organized as follow: first, the extension is presented in Sec. II, then some factors influencing the quality of outdoor EL imaging are presented and studied in Sec. III. One of those factors, the effect of motion between images of a set, is further analyzed in Sec. IV, before a discussion of the results and presentation of planned future work in Sec. V.

\section{SNR $\mathrm{AVG}_{\mathrm{A}}$ : THE PROPOSED EXTENSION TO SNR 50}

Even though the standard does not explain in depth the formula for $\mathrm{SNR}_{50}$, it can be understood in the following way. As the name indicates, a measure of signal to noise ratio contains two elements, the estimated power of the signal and that of the noise. In the $\mathrm{SNR}_{50}$ formula, the estimate of the signal is the average between the two EL images minus the background image. The noise term is the absolute difference between the two EL images, scaled by a constant.

Let us assume that $2|\mathrm{~N}|$ images have been acquired alternatingly: $|\mathrm{N}|$ of the panel with bias current $\left(E L_{i}\right)$ and $|\mathrm{N}|$ of the unpowered panel $\left(B G_{i}\right)$. The following equation is proposed as extension of $\mathrm{SNR}_{50}$ :

$$
S N R_{A V G}=\frac{\sum_{k}\left(\operatorname{avg}_{i \in N}\left(E L_{i}(k)-B G_{i}(k)\right)\right)}{\sum_{k}\left(\left|\operatorname{avg}_{i \in N_{1}}\left(E L_{i}(k)\right)-a v g_{j \in N_{2}}\left(E L_{j}(k)\right)\right| \cdot K\right)}
$$


with $K=\sqrt{0.5} \cdot\left(\frac{2}{\pi}\right)^{-0.5}$ (as for the $\mathrm{SNR}_{50}$ measure), $\mathrm{N}_{1}$ representing the odd numbers between 1 and $|\mathrm{N}|$, and $\mathrm{N}_{2}$ the even ones.

\section{FACTORS INFLUENCING THE QUALITY}

In order to evaluate the performance of the proposed use of multiple images and the measure, the dataset from [7] is used. It consists of 31 sets of 100 images (50 biased and 50 background images for each set, acquired alternatively). Each set was acquired outdoor at various GHI levels ranging from $668 \mathrm{~W} \mathrm{~m}^{-2}$ to $927 \mathrm{~W} \mathrm{~m}^{-2}$. The acquisition system consisted in an InGaAs camera Hamamatsu model C12741-03, and an OD>4.0 $1150 \mathrm{~nm}$ band-pass filter with $50 \mathrm{~nm}$ FWHM from Edmund Optics. The camera resolution is $640 \mathrm{x} 512$, although before computing the measure, the images were cropped to the region of interest (approximately 450 x 450 pixel). The PV panel imaged is a mechanically stressed 36 cell multicrystalline silicon solar panel with 1 x 1 meter dimension.

\section{A. Varying sun irradiance}

As shown in previous work [7], the average pixel value varies with the sun irradiance. Therefore, it is necessary to acquire alternatingly $E L$ and $B G$ images to obtain similar average value variation on both types (otherwise, the difference computed in the $\mathrm{SNR}_{\mathrm{AVG}}$ formula would account considerably for unwanted terms). For similar reasons, the extension of the noise part of the $\mathrm{SNR}_{\mathrm{AVG}}$ formula splits images between even and odd, therefore "interleaving" the images.

Fig. 1 shows two images with similar visual quality and SNR $_{\text {AVG }}$ acquired at different GHI. The images presented in this section are scaled between [-3, 47] (corresponding to the minimum and maximum $1 / 1000$ of the dataset at $671 \mathrm{~W} \mathrm{~m}^{-2}$ ).

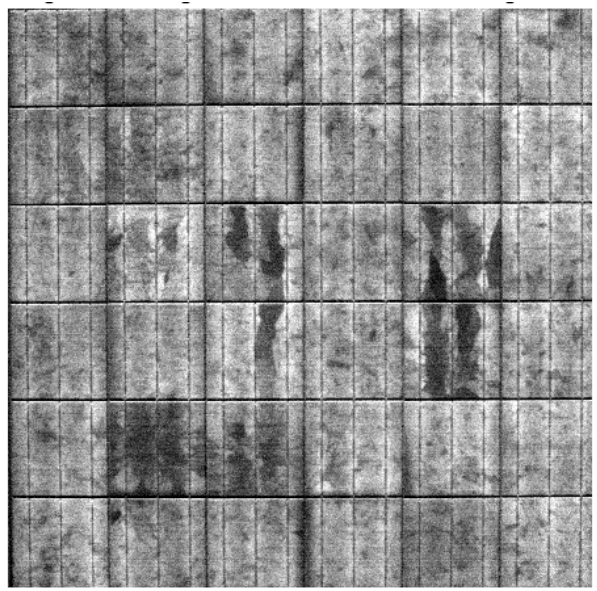

$\mathrm{SNR}_{\mathrm{AVG}}=6.05$,

$\mathrm{GHI}=671 \mathrm{~W} \mathrm{~m}^{-2}$

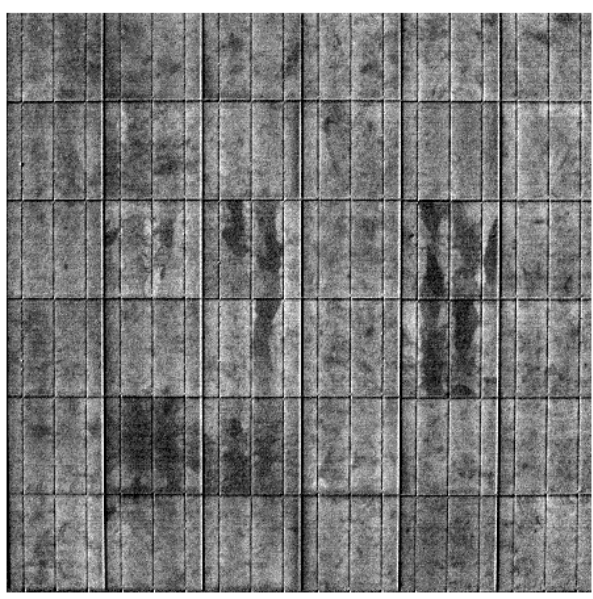

$\mathrm{SNR}_{\mathrm{AVG}}=5.96$,

$\mathrm{GHI}=815 \mathrm{~W} \mathrm{~m}^{-2}$

Fig. 1 Average difference images at two GHI

\section{B. Number of images acquired}

Another important factor for the quality level is the number $|\mathrm{N}|$ of images acquired of both types (biased and unpowered module). The impact of the number of images over the $\mathrm{SNR}_{\mathrm{AVG}}$ value is illustrated in Fig. 2. It shows that the measure increases with the increase of number of images used.

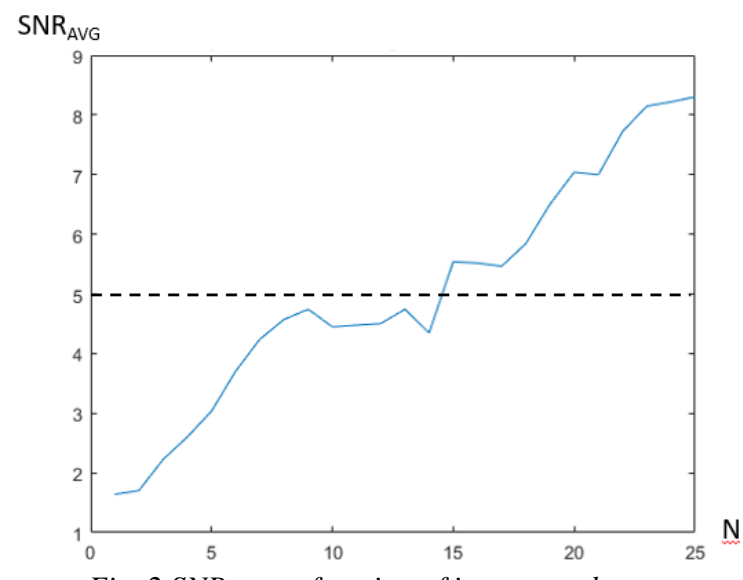

Fig. 2 SNRAVG as function of image number (acquired at $\mathrm{GHI}=685 \mathrm{~W} \mathrm{~m}^{-2}$ )

This is furthermore illustrated in Fig. 3 by displaying the difference between the average over $|\mathrm{N}| E L$ and $|\mathrm{N}| B G$ images and the corresponding $\mathrm{SNR}_{\mathrm{AVG}}$ values. 


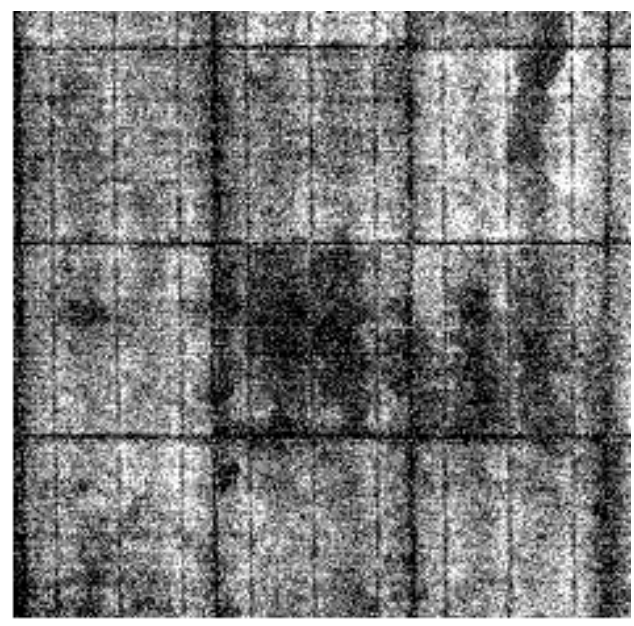

$\mathrm{SNR}_{\mathrm{AVG}}=3.04,5$ images

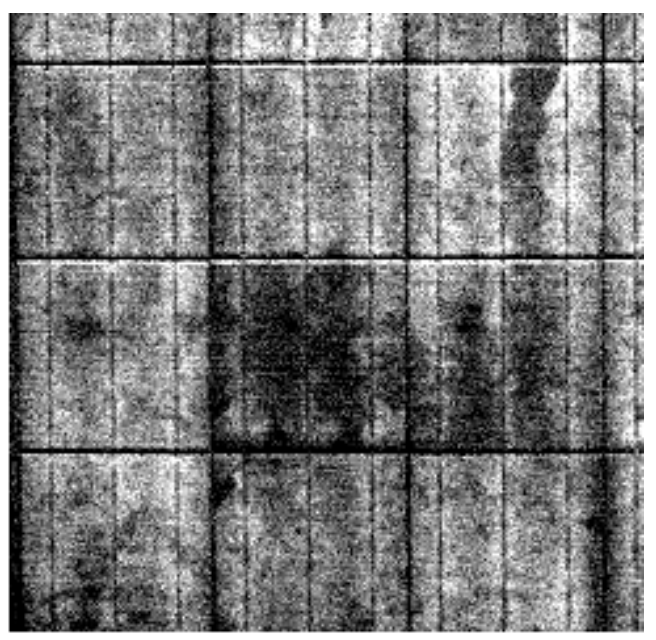

$\mathrm{SNR}_{\mathrm{AVG}}=4.45,10$ images

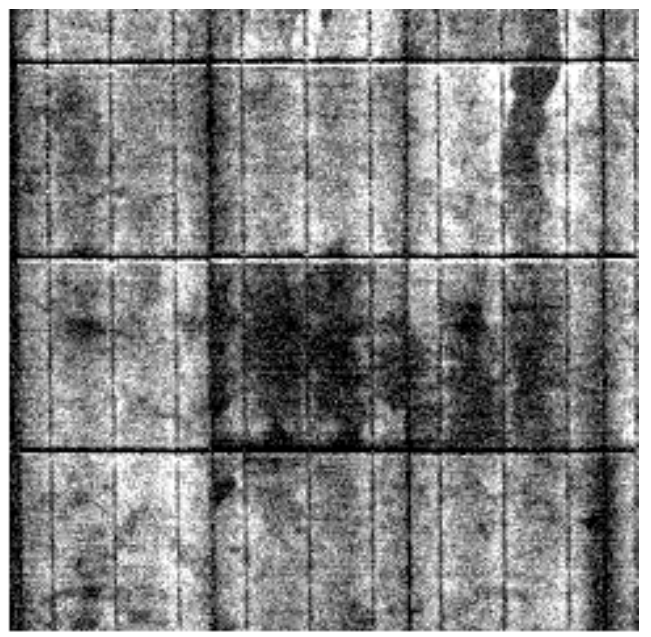

$\mathrm{SNR}_{\mathrm{AVG}}=5.54,15$ images

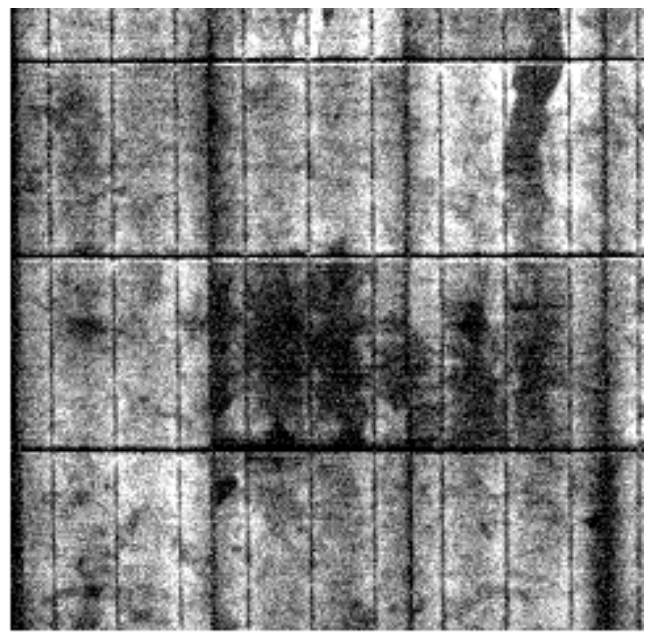

$\mathrm{SNR}_{\mathrm{AVG}}=7.04,20$ images

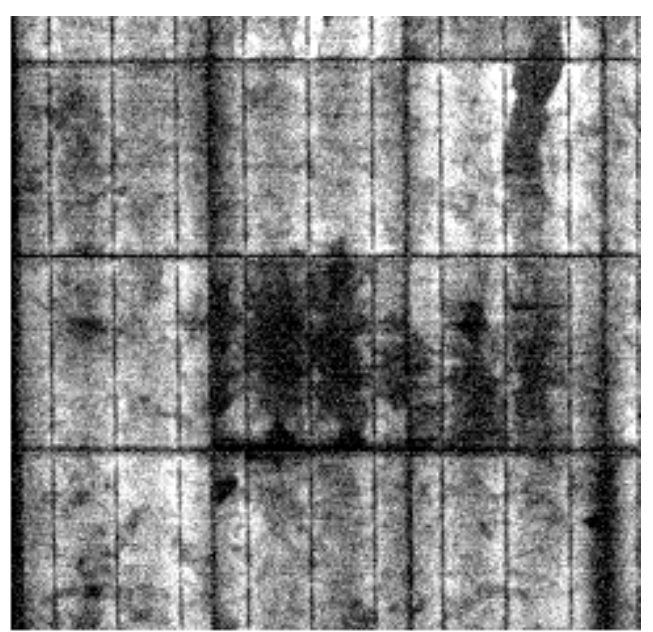

$\mathrm{SNR}_{\mathrm{AVG}}=8.3,25$ images

Fig. 3 Part of a difference image using various number of images (acquired at $\mathrm{GHI}=685 \mathrm{~W} \mathrm{~m}^{-2}$ )

The tendency pictured in Fig. 3 is visible on all datasets: the measured SNR $_{\mathrm{AVG}}$ increases with the number of averaged images. The increase in image quality for daylight EL imaging is crucial to the identification of faults with small dimensions, such as micro-cracks, fingers interruptions, among others. Assumably for this reason, [4] establishes that the minimum acceptable SNR for EL outdoor measurements is $\geq 5$.

\section{Noise Estimation}

For an additive noise, the simple model is:

$$
g=f+n
$$

where $g$ is the observed image, $f$ the original "noise free" image and $n$ an additive noise. If we consider that averaging over the $|\mathrm{N}|$ biased images yields a good estimation of $f$, then an estimate of the noise can be computed as 


$$
n=g(i)-\operatorname{mean}_{i}(g(i))
$$

where $i$ is the image index (i $\in[1,|\mathrm{~N}|]$ ) and mean $_{i}(g(i)$ ) represents the estimation of the real image. The validity of this equation depends mainly on two points: how good is the "clean image" estimation $\operatorname{mean}_{i}(g(i))$ and whether we can consider that the noise is purely additive. This section focuses on the validity of the estimated noise image.

Using projections of each noise image onto the $\mathrm{X}$ and $\mathrm{Y}$ axes, it is possible to evaluate how accurate the estimation is. Fig. 4 illustrates such a noise image and the corresponding sum of squared pixel respectively over the $\mathrm{X}$ and $\mathrm{Y}$ axes. It is visible in both the image and the projection on the $\mathrm{Y}$ axis that some of the panel structure is still present. One probable origin of those patterns is camera motion between some of the images from the same set. As the presence of such structure has been noted in many noise images (almost all of the 31 sets $x 50$ biased images), a study on the role of motion inside a set of images is presented in the next section.
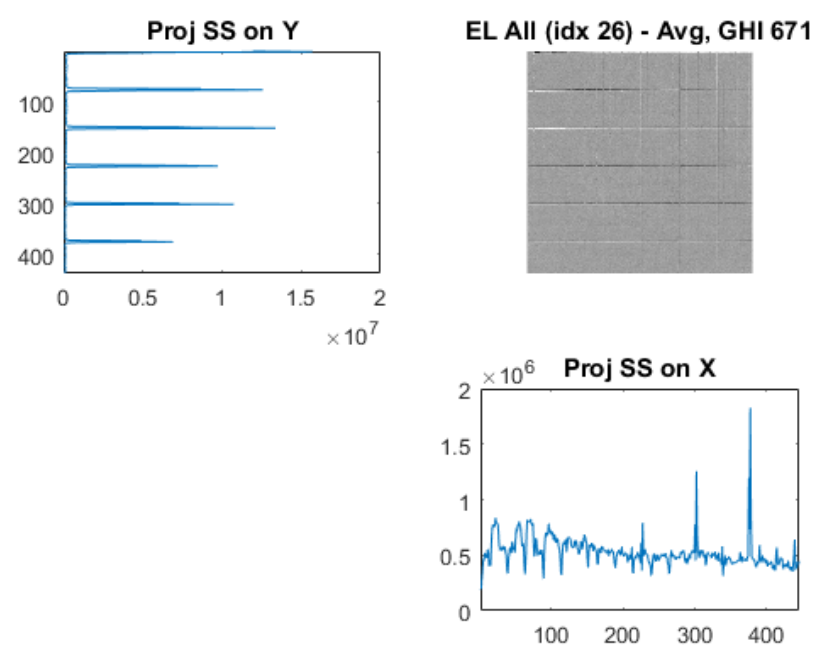

Fig. 4 Estimated noise image (upper right corner) and corresponding sum of squared pixel values on $X$ and $Y$ axes

\section{Motion Estimation AND SELECTION}

As the final aim is to apply the processing to images captured by a camera mounted on a UAV, camera motion is inevitable.

\section{A. Process applied}

For each of the 31 sets of images, the first stage of the motion estimation process is to choose which of the 50 acquired images will serve as the reference to estimate motion against. It is done by selecting the image which is the closest (in the mean squared error sense) to the average image. Then for each of the 49 other images, the motion is estimated using the method presented by Chan et al. in [9]. As this method estimates motion with subpixel accuracy, it is not possible to compensate for all of it without some kind of interpolation. The first approach used here is then to select only the images that are "close enough" to the reference one. This is determined using a threshold on the maximum amplitude of the computed displacement (called motion vector). In this paper, only images with an estimated motion smaller than 0.05 pixels (i.e. around $0.1 \mathrm{~mm}$ ) were kept.

\section{$B$. Influence on the quality and noise}

Fig. 5 presents the obtained difference image based on averages for one of the sets of images (at GHI $671 \mathrm{~W} \mathrm{~m}^{-2}$, i.e. the same set used for Fig. 4). As explained in the previous section, for this paper a threshold of 0.05 pixel on the maximum estimated displacement relatively to the average image is chosen. Practically for this set, 23 images, over the 50 available ones, are selected as having a motion estimation (i.e. displacement) smaller than 0.05 towards the average image. Fig. 5 illustrates the effect of selection by motion over the obtained quality by presenting for comparison the average difference image over 23 randomly chosen images (left) as opposed to the 23 images selected as "close enough" to the reference one.
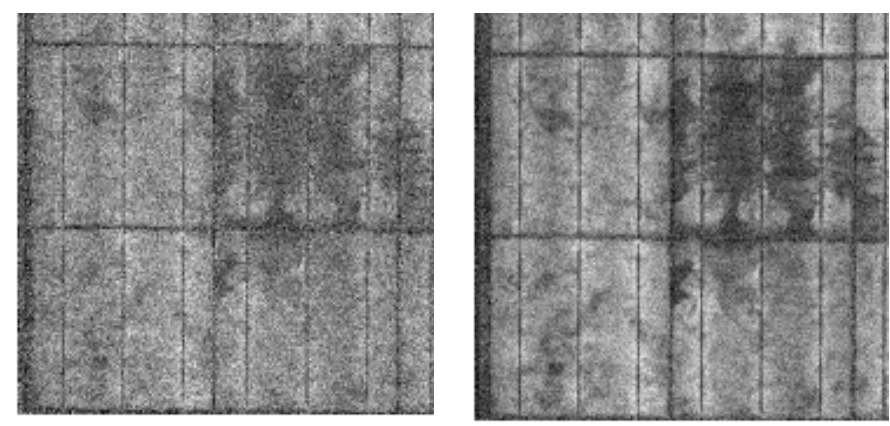

Fig. 5 Example of the effect of selection by motion on a portion of average difference image: using 23 random images (left, $S N R_{A V G}=4.2$ ) or using 23 motion selected images (right, $\left.S N R_{A V G}=4.7\right)$

The effect on the noise image is illustrated in Fig. 6, which is similar to Fig. 4, except that the average image is computed on the 19 motion selected images only. In comparison to Fig. 4, the amount of structure present in Fig. 6 is significantly decreased. It is clearly visible in the projections onto the $\mathrm{X}$ and $\mathrm{Y}$ axes, which are about an order of magnitude smaller with the selection.

As illustrated in Fig. 5 and Fig. 6, by selecting carefully the images used to average, it is possible to obtain better quality than by using the same number of image randomly selected. This is shown both on the visual evaluation in Fig. 5 and quantitatively on Fig. 6. 

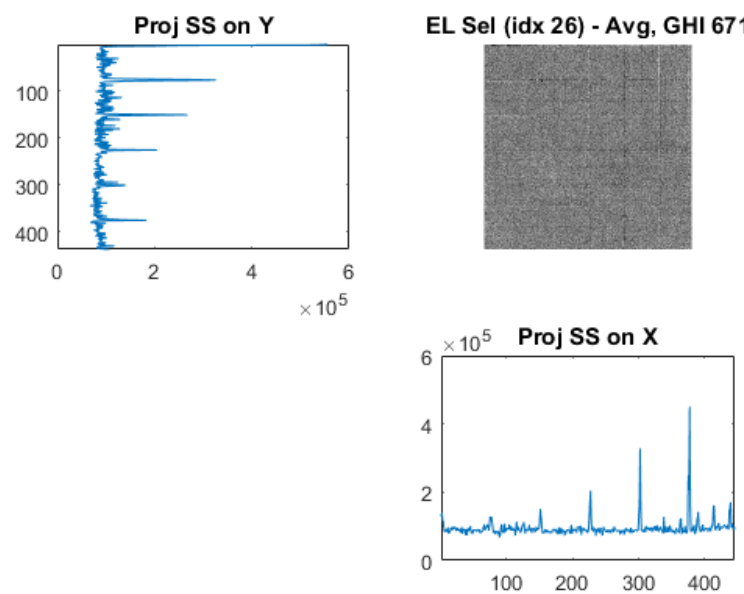

Fig. 6 Estimated noise image and corresponding sum of squared pixel values on $X$ and $Y$ axes for the same image as in Fig. 4

\section{DISCUSSION AND FUTURE WORK}

In this paper, we study some factors influencing the quality of EL images and present the $\mathrm{SNR}_{\mathrm{AVG}}$, an extension to the existing $\mathrm{SNR}_{50}$ measure to the case of multiple images. Results show that averaging over several images improves the quality both visually and by the $\mathrm{SNR}_{\mathrm{AVG}}$. In the context of outdoor EL imaging, it is also recommended to acquire the EL and BG images alternatingly to account for varying sun intensity. Furthermore, it is shown that using motion estimation for selecting images can improve the visual and measured quality. Finally, this paper illustrates that various $\mathrm{SNR}_{\mathrm{AVG}}$ levels correspond to the visual visibility of different defect types, and an extension would be to establish a more direct correspondence.

\section{REFERENCES}

[1] J. B. S. Koch, T. Weber, C. Sobottka, A. Fladung, P. Clemens, "Outdoor Electroluminescence Imaging of Crystalline Photovoltaic Modules: Comparative Study between Manual Ground-Level Inspections and DroneBased Aerial Surveys,” 32nd Eur. Photovolt. Sol. Energy Conf. Exhib., pp. 1736-1740, 2016.

[2] M. Köntges et al., "Review of Failures of Photovoltaic Modules,” IEA-Photovoltaic Power Syst. Program., pp. 1140, 2014.

[3] A. "Goldeye CL-033," https://www.alliedvision.com/en/products/cameras/detail/Go ldeye/CL-033 TEC1.html. .

[4] IEC, "PNW/TS 82-901 Ed. 1.0 Photovoltaic devices - Part 13: Electroluminescence of photovoltaic modules (proposed future IEC TS 60904-13).” http://www.iec.ch/.

[5] L. Stoicescu, M. Reuter, and J. . Werner, "Daysy: Luminescence Imaging of PV Modules in Daylight,” 29th Eur. Photovoltaics Sol. Energy Conf. Exhib. Amsterdam, Netherlands, pp. 2553-2554, 2014.

[6] T. J. Silverman, M. G. Deceglie, K. Vansant, S. Johnston, and I. Repins, "Illuminated outdoor luminescence imaging of photovoltaic modules," IEEE Photovolt. Spec. Conf., 2017.

[7] G. A. dos Reis Benatto et al., "Luminescence imaging strategies for drone-based PV array inspection,” 33rd Eur. Photovolt. Sol. Energy Conf. Exhib., pp. 2016-2020, 2017.

[8] J. Adams et al., "Non-Stationary Outdoor EL-Measurements with a Fast and Highly Sensitive InGaAs Camera," 32nd Eur. Photovolt. Sol. Energy Conf. Exhib., 2016.

[9] S. H. Chan et al., "Sub-pixel motion estimation without interpolation”, Proceedings of ICASSP. 2010 